\title{
Calculating interaction energies in transition metal complexes with local electron correlation methods
}

\author{
J. Grant Hill and James A. Platts ${ }^{\text {a) }}$ \\ School of Chemistry, Cardiff University, Park Place, Cardiff CF10 3AT, United Kingdom
}

(Received 2 July 2008; accepted 25 August 2008; published online 1 October 2008)

\begin{abstract}
The results of density fitting and local approximations applied to the calculation of transition metal-ligand binding energies using second order Møller-Plesset perturbation theory are reported. This procedure accurately reproduces counterpoise corrected binding energies from the canonical method for a range of test complexes. While counterpoise corrections for basis set superposition error are generally small, this procedure can be time consuming, and in some cases gives rise to unphysical dissociation of complexes. In circumventing this correction, a local treatment of electron correlation offers major efficiency savings with little loss of accuracy. The use of density fitting for the underlying Hartree-Fock calculations is also tested for sample Ru complexes, leading to further efficiency gains but essentially no loss in accuracy. (C) 2008 American Institute of Physics.
\end{abstract}

[DOI: $10.1063 / 1.2982790]$

\section{INTRODUCTION}

The computational resources required for correlated post-Hartree-Fock (HF) electronic structure calculations, in general, scale poorly with molecular size, limiting application of conventional methods to relatively small species. Recent developments in density fitting (DF) and local electron correlation methods have improved this scaling dramatically, thus requiring much less computational resource and in favorable cases approach linear scaling with molecular size.

The DF approximation expands orbital product densities in an optimized auxiliary DF basis set. First used in density functional theory, recent work has demonstrated its utility in $\mathrm{HF}$ and correlated $a b$ initio methods, notably second order Møller-Plesset perturbation theory (MP2), and coupledcluster methods. ${ }^{1-3}$ Local correlation methods exploit the rapid fall-off of electron-electron repulsion with distance by localizing the canonical molecular orbitals and restricting excitation to domains spatially close to the resulting orbitals. ${ }^{4-8}$ This yields significant efficiency gains, and the construction also effectively eliminates basis set superposition error (BSSE) from the correlation energy., ${ }^{9,10}$

Methods using both of these approximations together, such as density fitted local MP2 (DF-LMP2), eliminate many of the bottlenecks associated with conventional post-HF $a b$ initio calculations. ${ }^{11}$ Demonstrations of the power of this approach are increasingly common, with applications in many areas of chemistry. ${ }^{12-21}$ Recently, DF-LMP2 has been shown to produce excellent results for weak, noncovalent interactions such as hydrogen bonding and stacking interactions at a fraction of the cost of conventional methods. ${ }^{20-22}$ Coupled with spin-component scaling (SCS) ${ }^{23}$ this method compares well with benchmark coupled-cluster data. ${ }^{20,24,25}$ The efficiency of this approach has allowed application to larger sys-

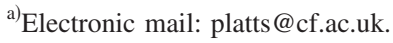

tems than are feasible with canonical MP2, such as nucleic acid base-pair steps. ${ }^{21}$

Correlation consistent basis sets, originally developed by Dunning $^{26}$ and denoted cc-pVnZ, converge systematically to the basis set limit. These basis sets have thus found extensive use in post-HF calculations. Balabanov and Peterson ${ }^{27}$ recently published cc-pVnZ basis sets for the first row transition metal (TM) elements. DF methods require an auxiliary basis set in which to expand orbital product densities: such auxiliary basis sets are well established for most of the $p$-block elements and are in widespread use. ${ }^{28,29}$ Following this work, auxiliary basis sets for the $3 d$ TMs were developed and were shown to produce relative errors in correlation energies of a test set of molecules that were of the order of $0.002 \%{ }^{30}$

In this paper, the performance of DF-LMP2 for calculation of binding energies of ligands to a range of TMs is tested. While previous results indicate that total correlation energies of TM complexes are well reproduced by the DF approximation with correlation consistent auxiliary basis sets, ${ }^{30}$ relative energies remain untested. Moreover, local methods that work well for noncovalent interactions may not describe metal-ligand bonds and their rupture with sufficient accuracy. Our chief interest lies in metals binding to nucleic acids, in which large sections of DNA act as ligands to metals such as platinum, ruthenium, or rhodium. TMs are also vital components in enzymes, homogeneous catalysts, and functional materials, to name but a few areas of application. The ability to calculate accurate $a b$ initio ligand binding energies using DF-LMP2 would be of potential benefit in many areas of chemistry, for instance, in benchmarking more approximate methods such as density functional theory.

We have therefore drawn up a set of complexes with which to test these methods, including early (Ti), middle (Fe, $\mathrm{Ru}$ ), and late (Ni) TMs, oxygen, nitrogen, and halide ligands, and charged as well as neutral species (see Fig. 1). All complexes are stable as singlet species and should be well de- 


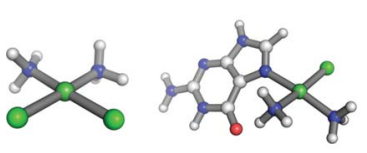

1

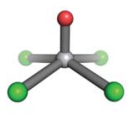

4

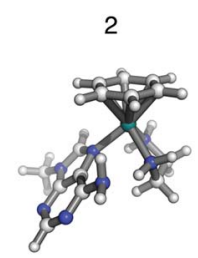

5

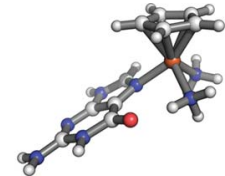

3

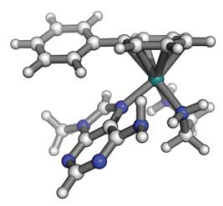

6
FIG. 1. (Color online) Complexes studied in this investigation: (1) $\mathrm{NiCl}_{2}\left(\mathrm{NH}_{3}\right)_{2}$; (2) $\mathrm{NiCl}\left(\mathrm{NH}_{3}\right)_{2}$, guanine; (3) $\mathrm{Fe}(\mathrm{NH} 3)_{2} \mathrm{Cp}$, guanine; (4) $\left[\mathrm{TiCl}_{4} \mathrm{O}\right]^{2-} ;$ (5) $\left[\left(\eta^{6}\right.\right.$-benzene) $\left.\mathrm{Ru}^{\mathrm{II}}(\mathrm{en})-\mathrm{Cl}\right]$, adenine; (6) $\left[\left(\eta^{6}\right.\right.$-biphenyl) $\left.\mathrm{Ru}^{\mathrm{II}}(\mathrm{en})-\mathrm{Cl}\right]$, adenine.

scribed by a single Slater determinant, such that MP2 and underlying HF treatments should provide reasonable approximations to their electronic structure. Tests on such small complexes are required in order for comparison with traditional MP2 and/or coupled-cluster data. If successful, we envisage application of this DF-LMP2 method to determine the binding of potential anticancer agents such as cisplatin to DNA and as the quantum mechanical method within hybrid quantum mechanical/molecular mechanics studies of metalDNA adducts.

\section{COMPUTATIONAL PROCEDURE}

All of the gas-phase geometries used in this investigation were obtained with the GAUSSIAN 03 package ${ }^{31}$ using the B3LYP/TZVP level of theory, with the exception of the Ru containing complexes 5 and 6 which were taken from the supporting information of Ref. 32. Subsequent single point energy calculations were performed with the MOLPRO package of $a b$ initio programs $^{33}$ using the MP2, DF-MP2, DF-LMP $2,{ }^{11}$ and DF-LCCSD(T0) ${ }^{6,34-37}$ methods. In the latter, (T0) indicates that local perturbative triples were calculated, full details on how this differs from the more common (T) perturbative triples can be found in Refs. 34 and 35 . Calculations that compensated for BSSE did so with the counterpoise (CP) method of Boys and Bernardi. ${ }^{38}$ All of the interaction energies use rigid geometries, such that when the bond is broken both fragments retain the same geometries they had in the bound system.

The atomic orbital (AO) basis sets employed in this investigation were the aug-cc-pVTZ-NR first row TM sets of Balabanov and Peterson, ${ }^{27}$ the aug-cc-pVTZ-PP basis set and pseudopotential for $\mathrm{Ru}$ of Peterson et al. ${ }^{39}$ and the aug-ccpVTZ sets of Kendall et al. ${ }^{40}$ on all other atoms. In an effort to decrease computational cost and the residual HF BSSE in local correlation calculations some energies were evaluated using the cc-pVTZ basis set on hydrogen atoms. In the following text the full aug-cc-pVTZ basis sets are referred to as AVTZ, and the sets where cc-pVTZ is used for $\mathrm{H}$ are referred to as AVTZ* DF in the MP2 case was carried out with the corresponding aug-cc-pVTZ and cc-pVTZ MP2 fitting basis sets. ${ }^{28,30} \mathrm{DF}$ for LCCSD(T0) used the aug-cc-pVTZ and ccpVTZ MP2 fitting basis sets for integrals with up to two external orbitals and the aug-cc-pVQZ and cc-pVQZ MP2 fitting basis sets for integrals over three and four external orbitals, with the exception of $\mathrm{Ni}$ which used the aug-ccpVTZ MP2 fitting basis as larger auxiliary basis sets are not currently available for this atom.

The localized orbitals required for LMP2 and $\operatorname{LCCSD}(\mathrm{T} 0)$ treatments were determined via the method of Pipek and Mezey, ${ }^{41}$ with the contribution of the two most diffuse functions removed from the criteria by setting the corresponding overlap matrix rows and columns to zero. The subsequent domain selection used the procedure of Boughton and Pulay, ${ }^{42}$ with a completeness criterion of 0.985 . For systems involving $\pi$-orbitals and ring structures the relevant domains were merged to ensure the correlation energy was invariant to unitary transformations among those orbitals. SCS MP2 is employed to investigate if it produces interaction energies close to those from DF-LCCSD(T0) using the original parameters of Grimme. ${ }^{43}$

To enable DF-MP2 calculations on complexes that contain the Ru atom appropriate auxiliary MP2 fitting bases were constructed. These sets were optimized for use with the cc-pVnZ-PP and aug-cc-pVnZ-PP (where $n=\mathrm{D}, \mathrm{T}, \mathrm{Q}$, and 5) orbital basis sets of Peterson et al. ${ }^{39}$ using analytic gradients for the optimization of auxiliary basis sets ${ }^{29}$ implemented in the RICC2 module ${ }^{2,44}$ of the TURBOMOLE package. ${ }^{45,46}$ The construction and optimization followed the procedure outlined in Ref. 30 with the exception that the extra diffuse function for each angular momentum quantum number in the augmented sets was optimized on top of the standard basis, instead of the whole auxiliary basis set being reoptimized. In order to account for the different oxidation states encountered in TM complexes the criteria used to determine if the auxiliary basis set constructed is sufficiently accurate is that the mean absolute error and the SD of the error due to the DF approximation over a representative test set of molecules are at least two orders of magnitude smaller than the error due to orbital basis set incompleteness. The latter is defined in the current case as the relative change in correlation energy when the AO basis set size is increased to the next cardinal number in the series, for example, the change in energy when a cc-pVQZ-PP basis set is used instead of the cc-pVTZ-PP basis. As only the aug-cc-pVTZ-PP basis is employed further within this investigation only the cc-pVTZ-PP and aug-ccpVTZ-PP auxiliary basis sets are discussed here, further details on the remaining sets can be obtained from the EPAPS. $^{47}$

The DF approximation and orbital basis set incompleteness errors were evaluated for five simple complexes, namely, RuF, $\mathrm{RuO}, \mathrm{RuO}_{2}, \mathrm{RuO}_{4}$, and $\mathrm{Ru}(\mathrm{CO})_{5}$. The relative percentage error in the $\mathrm{DF}$ is given by $\Delta_{i}^{\mathrm{DF}}$ $=\left(E^{\text {corr }}-E^{\mathrm{DF}-\text { corr }}\right) / E^{\text {corr }} \times 100 \%$, where $E^{\mathrm{DF}-\text { corr }}$ and $E^{\text {corr }}$ are the DF and conventional MP2 correlation energies, respectively. Similarly the relative percentage error in the orbital basis is given by $\Delta_{i}^{\mathrm{AO}}=\left(E^{\text {large basis }}-E^{\text {small basis }}\right) / E^{\text {large basis }}$ $\times 100 \%$. This leads to a mean absolute percentage error (MAE) of 0.0030 and a SD of 0.0012 in the DF of the ccpVTZ-PP basis, to be compared with an MAE of 8.1527 and a SD of 1.7742 for the orbital basis set incompleteness. For the aug-cc-pVTZ-PP basis there is a MAE and SD of 0.0022 
TABLE I. Interaction energies $\left(\mathrm{kcal} \mathrm{mol}^{-1}\right)$ for the removal of ligands from the $\mathrm{Ni}$ complexes 1 and 2 . See text for further details.

\begin{tabular}{lccc}
\hline \hline & \multicolumn{3}{c}{ System } \\
\cline { 2 - 4 } Method & $1 \mathrm{a}$ & $1 \mathrm{~b}$ & $\mathbf{2}$ \\
\hline MP2/AVTZ & -40.42 & -158.79 & -84.88 \\
CP-MP2/AVTZ & -39.00 & -156.76 & -82.18 \\
DF-MP2/AVTZ & -40.43 & -158.80 & -84.88 \\
CP-DF-MP2/AVTZ & -39.00 & -156.77 & -82.18 \\
DF-LMP2/AVTZ & -38.62 & -157.19 & -82.65 \\
SCS/AVTZ & -34.48 & -152.42 & -78.93 \\
DF-LMP2/AVTZ* & -38.54 & -157.06 & -81.99 \\
SCS/AVTZ* & -34.41 & -152.31 & -78.25 \\
DF-LCCSD(T0)/AVTZ* & -32.14 & -153.27 & $\ldots$ \\
\hline \hline
\end{tabular}

and 0.0014, respectively, in the DF and of 7.1318 and 1.6202 in the orbital basis set. These statistics indicate that the DF approximation introduces an insignificant error in both cases, not only in comparison to the orbital basis set incompleteness, where the error is three orders of magnitude smaller, but also in terms of the change in overall correlation energy [see EPAPS (Ref. 47)]. It should be noted that as the test systems included non-Ru atoms the error analysis presented does include some contribution from the cc-pVTZ and augcc-pVTZ orbital and auxiliary basis sets for those atoms. However, this also means that the tests should be indicative of the magnitude of errors that can be expected in applications of MP2 density fitting with Ru containing complexes. Analogous measures of the errors due to the DF approximation and orbital basis incompleteness for the remaining six basis sets are presented in the supporting EPAPS. ${ }^{47}$ While the error due to the orbital basis set decreases as the number of functions is increased and the basis set becomes more flexible, the relative DF error in using the equivalent auxiliary basis set is also reduced such that it is always at least three orders of magnitude smaller.

\section{RESULTS AND DISCUSSION}

Table I reports interaction energies, with no account of relaxation energies, for the removal of the $\mathrm{NH}_{3}$ and $\mathrm{Cl}^{-}$ ligands from complex 1 , which have been labeled as systems $1 \mathrm{a}$ and $1 \mathrm{~b}$, respectively, and for the removal of the guanine ligand from complex 2. As expected the results indicate that the DF approximation works well in this case and the difference between the DF-MP2 interaction energies both with and without the CP correction applied illustrates that the BSSE is small in all three cases, at approximately $2 \mathrm{kcal} \mathrm{mol}^{-1}$. Comparison of the DF-LMP2/AVTZ data with that of CP corrected MP2 indicates that the local correlation treatment performs well in these cases, producing interaction energies within $0.5 \mathrm{kcal} \mathrm{mol}^{-1}$ of the $\mathrm{CP}$ corrected canonical case. This suggests that while the BSSE in the systems is small, the LMP2 treatment is effective at eliminating it without the need for potentially expensive $\mathrm{CP}$ corrections. It also indicates that the orbital localization algorithm and domain selection criteria employed are capable of properly describing the TM center within the LMP2 construction.

Omitting the diffuse functions on the hydrogen atoms in the case of the AVTZ* basis produces a very small change in the DF-LMP2 interaction energy, while producing a noticeable reduction in computational cost (as a rough indication, the AVTZ* calculation on complex 2 only required approximately $60 \%$ of the calculation time for AVTZ, with both computations run on the same AMD Opteron 246 based machine). Previous investigations have shown that such basis sets also help to minimize the amount of residual HF BSSE in interaction energies. ${ }^{20,22,48}$ Higher accuracy interaction energies for complex 1 were calculated at the DF-LCCSD(T0) level of theory and MP2 overestimates the interaction energy by 6.4 and $3.8 \mathrm{kcal} \mathrm{mol}^{-1}$ for systems $1 \mathrm{a}$ and $1 \mathrm{~b}$, respectively. SCS of the DF-LMP2 energies produces interaction energies that are in better agreement with those produced by the local coupled-cluster method, with errors of 2.27 and $0.96 \mathrm{kcal} \mathrm{mol}^{-1}$ in $1 \mathrm{a}$ and $1 \mathrm{~b}$, respectively. SCS interaction energies for system 2 are included for completeness, it can be seen that SCS shows the guanine ligand to be around 3-4 $\mathrm{kcal} \mathrm{mol}^{-1}$ less strongly bound than with unscaled MP2 methods. The DF-LCCSD(T0) results presented in Table I treated all pairs within the local construction as strong pairs. By employing the default distance based criteria for the selection of pair classes, where some pairs are treated at the MP2 level (see Ref. 49 for a detailed discussion on the selection of local correlation domains), the interaction energies for systems $1 \mathrm{a}$ and $1 \mathrm{~b}$ become -33.74 and $-153.86 \mathrm{kcal} \mathrm{mol}^{-1}$, respectively. This is accompanied by a large reduction in execution time for the calculation of the correlation energy: $57.6 \%$ in the case of $1 \mathrm{a}$ and $52.7 \%$ for $1 \mathrm{~b}$.

Table II shows that the interaction energies for the removal of the guanine (3a), $\mathrm{NH}_{3}(3 \mathrm{~b}), \mathrm{Cl}(3 \mathrm{c})$, and cyclopentadienyl (3d) ligands from complex 3 again indicate that the BSSE is small with values between 1.9 and $4.3 \mathrm{kcal} \mathrm{mol}^{-1}$. A comparison of the $\mathrm{CP}$ corrected energies both with and without the DF approximation also indicates that the combi-

TABLE II. Interaction energies $\left(\mathrm{kcal} \mathrm{mol}^{-1}\right)$ for the removal of various ligands from the Fe complex 3 and the Ti complex 4. See text for further details.

\begin{tabular}{lcccccc}
\hline \hline & \multicolumn{7}{c}{ System } \\
\cline { 2 - 7 } Method & $3 \mathrm{a}$ & $3 \mathrm{~b}$ & $3 \mathrm{c}$ & $3 \mathrm{~d}$ & $4 \mathrm{a}$ & $4 \mathrm{~b}$ \\
\hline MP2/AVTZ & -62.23 & -25.53 & -133.28 & -187.25 & -370.07 & 39.54 \\
DF-MP2/AVTZ & -62.24 & -25.53 & -133.29 & -187.27 & -370.10 & 39.53 \\
CP-MP2/AVTZ & -58.72 & -23.59 & -130.51 & -183.00 & -376.12 & 41.30 \\
CP-DF-MP2/AVTZ & -58.72 & -23.59 & -130.52 & -183.01 & -378.69 & 41.30 \\
DF-LMP2/AVTZ & -58.40 & -22.81 & -130.89 & -183.20 & -368.77 & 40.77 \\
\hline \hline
\end{tabular}


TABLE III. Comparison of interaction energies $\left(\mathrm{kcal} \mathrm{mol}^{-1}\right)$ for the removal of adenine from the $\mathrm{Ru}$ arene complexes 5 and 6 .

\begin{tabular}{lcc}
\hline \hline & \multicolumn{2}{c}{ System } \\
\cline { 2 - 3 } Method & 5 & 6 \\
\hline HF & -45.80 & $\ldots$ \\
DF-HF & -45.80 & -39.13 \\
MP2 & -85.72 & $\ldots$ \\
DF-MP2 & -85.74 & $\ldots$ \\
DF-MP2//DF-HF & -85.72 & -86.79 \\
CP-DF-MP2//DF-HF & -80.79 & -80.24 \\
DF-LMP2 & -80.69 & $\ldots$ \\
DF-LMP2//DF-HF & -80.67 & -80.03 \\
\hline \hline
\end{tabular}

nation of orbital and auxiliary basis sets utilized produce a negligible error in the interaction energies. Using local correlation methods underestimates the $\mathrm{CP}$ energies by less than a kcal mol${ }^{-1}$ when the guanine and $\mathrm{NH}_{3}$ ligands are removed, while overestimation of around the same magnitude is observed with the $\mathrm{Cl}$ and cyclopentadienyl ligands. Once more this suggests that the LMP2 approach both removes the majority of the BSSE and is suitable for describing this type of TM complex.

Upon inspection of the interaction energies for the removal of the $\mathrm{O}^{2-}(4 \mathrm{a})$ and $\mathrm{Cl}^{-}$(4b) ligands from the Ti complex 4 in Table II it should be obvious that some serious problems were manifested in the $\mathrm{CP}$ calculation when removing $\mathrm{O}^{2-}$, highlighted by the fact that the interaction energy becomes more negative as the correction is applied. Inspection of the individual components of these calculations [included in the supporting EPAPS (Ref. 47)] shows that the $\mathrm{HF}$ calculation on $\mathrm{O}^{2-}$ in the full basis converges to a different state than if the smaller fragment basis is utilized. The $\mathrm{CP}$ corrected HF value is 0.126 a.u. (79.1 kcal mol${ }^{-1}$ ) lower in energy than the uncorrected value, a difference that is unrealistic in the context of the other data presented here. Further evidence for this can be seen in Table II as similar problems are not observed when a $\mathrm{Cl}^{-}$ligand is removed. Converging to a different state also has a large effect on the correlation energy but a cancellation of errors means that the $\mathrm{CP}$ values in Table II differ by only $6.1-8.6 \mathrm{kcal} \mathrm{mol}^{-1}$ when compared with the respective uncorrected energies. The small BSSEs exhibited in systems 1-3 (Tables I and II) suggest that the DF-LMP2 interaction energy for $4 \mathrm{a}$ is probably close to what a BSSE free energy would be. An advantage of local correlation methods over CP corrections is demonstrated as it is only the $\mathrm{HF}$ calculation for $\mathrm{O}^{2-}$ in the full basis where problems are manifested. The positive interaction energies shown for $4 \mathrm{~b}$ can be explained by the fact that breaking of this bond produces two negatively charged fragments. For reference, the B3LYP/TZVP interaction energy for the same system is $41.17 \mathrm{kcal} \mathrm{mol}^{-1}$.

Interaction energies for the removal of the adenine ligand from the $\mathrm{Ru}$ arene complexes 5 and 6 are presented in Table III. The MP2 and DF-MP2 energies for complex 5 differ by only $0.02 \mathrm{kcal} \mathrm{mol}^{-1}$, which indicates that the AVTZ auxiliary MP2 fitting basis set presented in this paper produces an accurate description of the correlation energy in systems of this type when combined with established AVTZ auxiliary sets on all other atoms. The initial self-consistent field calculation is a major bottleneck in the overall MP2 calculation for complex 5 (102389 s for the non-CP HF compared to $5161 \mathrm{~s}$ for the subsequent DF-LMP2 as an eight processor job on an SGI Altix 4700) and is likely to become prohibitive as the size of the arene in the complex is increased. DF-HF can reduce the time required for a HF calculation by around an order of magnitude. ${ }^{50,51}$ However, as there is currently no Ru atom AVTZ JK-fit basis for DF-HF available, the DF-HF results reported in Table III were carried out with the def2-QZVPP JK-fitting basis of Weigend ${ }^{52}$ on the Ru atom and AVTZ JK-fitting sets ${ }^{50}$ on all other atoms. The correlated calculations carried out on a DF-HF reference have been appended with the notation //DF-HF in Table III. It can be seen that the use of this basis for JK density fitting produces almost no change in the interaction energies relative to those computed with the conventional reference, with errors of no more than $0.02 \mathrm{kcal} \mathrm{mol}^{-1} \mathrm{ob}-$ served (the time taken for the DF-HF calculation was $9813 \mathrm{~s}$, using the same resources as above). Due to the negligible errors reported for system 5, the same JK-fitting basis for $\mathrm{Ru}$ is employed in the case of the larger complex 6 without further validation. For both complexes the LMP2 approximation produces an interaction energy that is very close to that evaluated at the CP corrected MP2 level. The error produced by the local correlation treatment is around $0.2 \mathrm{kcal} \mathrm{mol}^{-1}$, which is within chemical error of the more rigorous value and is small in comparison with the BSSE of 5-6 $\mathrm{kcal} \mathrm{mol}^{-1}$. Interaction energies for 5 and 6 calculated with the BHandH [as implemented in GAUSSIAN 03 (Ref. 31)] density functional theory have been reported as -79.31 and $-76.49 \mathrm{kcal} \mathrm{mol}^{-1}$, respectively. ${ }^{32}$ These data show that $\mathrm{BHandH}$ qualitatively predicts the correct trend of adenine binding, but results in a larger difference in interaction energy between the two systems than found with the more accurate DF-LMP2.

\section{CONCLUSIONS}

It has been demonstrated that DF-LMP2 reproduces conventionally calculated metal-ligand binding energies, with typical errors less than $1 \mathrm{kcal} \mathrm{mol}^{-1}$. Furthermore, CP corrections are rendered unnecessary by the use of local correlation methods; these corrections are generally small but require significant extra computations, and in one case leads to an unphysical description of the dissociated complex. Similar performance is observed across all complexes considered, for early/late and $3 d / 4 d$ TMs. For the smallest complex considered, DF-LCCSD(T0) data were also computed: this shows that MP2 methods overestimate binding, but that SCS brings MP2 predictions into line. Applications of these methods to the binding of potential anticancer agents to DNA are ongoing in our laboratory.

The MP2 auxiliary basis sets presented in this paper can be obtained from EPAPS (Ref. 47) and will be made available via the Environmental Molecular Sciences Laboratory Basis Set Exchange website ${ }^{53}$ (http://gnode2.pnl.gov/bse/ portal). 


\section{ACKNOWLEDGMENTS}

The authors would like to thank the EPSRC National Service for Computational Chemistry Software ${ }^{54}$ for the CPU time and access to the TURBOMOLE package that it provided.

${ }^{1}$ O. Vahtras, J. Almlöf, and M. W. Feyereisen, Chem. Phys. Lett. 213, 514 (1993).

${ }^{2}$ C. Hättig and F. Weigend, J. Chem. Phys. 113, 5154 (2000).

${ }^{3}$ M. Schütz and F. R. Manby, Phys. Chem. Chem. Phys. 5, 3349 (2003).

${ }^{4}$ P. Pulay, Chem. Phys. Lett. 100, 151 (1983).

${ }^{5}$ S. Saebø and P. Pulay, Annu. Rev. Phys. Chem. 44, 213 (1993).

${ }^{6}$ C. Hampel and H.-J. Werner, J. Chem. Phys. 104, 6286 (1996).

${ }^{7}$ M. Schütz, G. Hetzer, and H.-J. Werner, J. Chem. Phys. 111, 5691 (1999).

${ }^{8}$ G. Hetzer, M. Schütz, H. Stoll, and H.-J. Werner, J. Chem. Phys. 113, 9443 (2000).

${ }^{9}$ M. Schütz, G. Rauhut, and H.-J. Werner, J. Phys. Chem. A 102, 5997 (1998).

${ }^{10}$ N. Runeberg, M. Schütz, and H.-J. Werner, J. Chem. Phys. 110, 7210 (1999).

${ }^{11}$ H.-J. Werner, F. R. Manby, and P. J. Knowles, J. Chem. Phys. 118, 8149 (2003).

${ }^{12}$ T. van Mourik, Chem. Phys. Lett. 414, 364 (2005).

${ }^{13}$ T. Hrenar, G. Rauhut, and H.-J. Werner, J. Phys. Chem. A 110, 2060 (2006).

${ }^{14}$ R. A. Mata and H.-J. Werner, J. Chem. Phys. 125, 184110 (2006).

${ }^{15}$ L. F. Holroyd and T. van Mourik, Chem. Phys. Lett. 442, 42 (2007).

${ }^{16}$ L. Maschio, D. Usvyat, F. R. Manby, S. Casassa, C. Pisani, and M. Schütz, Phys. Rev. B 76, 075101 (2007).

${ }^{17}$ T. van Mourik, V. I. Danilov, E. Gonzalez, A. Deriabina, and V. I. Poltev, Chem. Phys. Lett. 445, 303 (2007).

${ }^{18}$ R. A. Mata, H.-J. Werner, S. Thiel, and W. Thiel, J. Chem. Phys. 128, 025104 (2008).

${ }^{19}$ E. Goll, T. Leininger, F. R. Manby, A. Mitrushchenkov, H.-J. Werner, and H. Stoll, Phys. Chem. Chem. Phys. 10, 3353 (2008).

${ }^{20}$ J. G. Hill, J. A. Platts, and H.-J. Werner, Phys. Chem. Chem. Phys. 8, 4072 (2006).

${ }^{21}$ J. G. Hill and J. A. Platts, Phys. Chem. Chem. Phys. 10, 2785 (2008).

${ }^{22}$ T. Takatani and C. D. Sherrill, Phys. Chem. Chem. Phys. 9, 6106 (2007).

${ }^{23}$ S. Grimme, J. Chem. Phys. 118, 9095 (2003).

${ }^{24}$ J. G. Hill and J. A. Platts, J. Chem. Theory Comput. 3, 80 (2007).

${ }^{25}$ J. Antony and S. Grimme, J. Phys. Chem. A 111, 4862 (2007).

${ }^{26}$ T. H. Dunning, J. Chem. Phys. 90, 1007 (1989).

${ }^{27}$ N. B. Balabanov and K. A. Peterson, J. Chem. Phys. 123, 064107 (2005).
${ }^{28}$ F. Weigend, A. Köhn, and C. Hättig, J. Chem. Phys. 116, 3175 (2002).

${ }^{29}$ C. Hättig, Phys. Chem. Chem. Phys. 7, 59 (2005).

${ }^{30}$ J. G. Hill and J. A. Platts, J. Chem. Phys. 128, 044104 (2008).

${ }^{31}$ M. J. Frisch, G. W. Trucks, H. B. Schlegel et al., GAussian 03, Revision C.01, Gaussian, Inc., Wallingford, CT, 2004.

${ }^{32}$ K. Gkionis, J. A. Platts, and J. G. Hill, Inorg. Chem. 47, 3893 (2008).

${ }^{33}$ MOLPRO, version 2006.4, a package of ab initio programs designed by H.-J. Werner, P. J. Knowles, R. Lindh, F. R. Manby, M. Schütz, P. Celani, T. Korana, A. Mitrushenkov, G. Rauhut, T. B. Adler, R. D. Amos, A. Bernhardsson, A. Berning et al.

${ }^{34}$ M. Schütz and H.-J. Werner, Chem. Phys. Lett. 318, 370 (2000).

${ }^{35}$ M. Schütz, J. Chem. Phys. 113, 9986 (2000).

${ }^{36}$ M. Schütz and H.-J. Werner, J. Chem. Phys. 114, 661 (2001).

${ }^{37}$ M. Schütz, Phys. Chem. Chem. Phys. 4, 3941 (2002).

${ }^{38}$ S. F. Boys and F. Bernardi, Mol. Phys. 19, 553 (1970).

${ }^{39}$ K. A. Peterson, D. Figgen, M. Dolg, and H. Stoll, J. Chem. Phys. 126, 124101 (2007).

${ }^{40}$ R. A. Kendall, T. H. Dunning, and R. J. Harrison, J. Chem. Phys. 96, 6796 (1992).

${ }^{41}$ J. Pipek and P. G. Mezey, J. Chem. Phys. 90, 4916 (1989).

${ }^{42}$ J. W. Boughton and P. J. Pulay, J. Comput. Chem. 14, 736 (1993).

${ }^{43}$ S. Grimme, Chem.-Eur. J. 10, 3423 (2004).

${ }^{44}$ C. Hättig, J. Chem. Phys. 118, 7751 (2003).

${ }^{45}$ R. Ahlrichs, M. Bär, M. Häser, H. Horn, and C. Kölmel, Chem. Phys. Lett. 162, 165 (1989).

${ }^{46}$ TURBOMOLE, Version 5.9.1, see http://www.cosmologic.de/ QuantumChemistry/main_turbomole.html

${ }^{47}$ See EPAPS Document No. E-JCPSA6-129-608838 for Gaussian exponents for the cc-pVnZ-PP and aug-cc-pVnZ-PP MP2-fitting auxiliary basis sets. $n=\mathrm{D}, \mathrm{T}, \mathrm{Q}$, and 5 in both cases. Also included are tables detailing the comparison of the number of GTOs in AO and auxiliary basis sets, relative percentage errors in basis set incompletness and from the DF approximation, correlation energies for the basis set test set, and HF and MP2 contributions to the interaction energy for $\mathrm{O}^{2-}$ removal from $\mathrm{TiCl}_{4} \mathrm{O}^{2-}$. For more information on EPAPS, see http://www.aip.org/ pubservs/epaps.html.

${ }^{48}$ O. Marchetti and H.-J. Werner, Phys. Chem. Chem. Phys. 10, 3400 (2008).

${ }^{49}$ H.-J. Werner and K. Pflüger, Annu. Rep. Comp. Chem. 2, 53 (2006).

${ }^{50}$ F. Weigend, Phys. Chem. Chem. Phys. 4, 4285 (2002).

${ }^{51}$ R. Polly, H.-J. Werner, F. R. Manby, and P. J. Knowles, Mol. Phys. 102, 2311 (2004)

${ }^{52}$ F. Weigend, J. Comput. Chem. 29, 167 (2008).

${ }^{53}$ K. L. Schuchardt, B. T. Didier, T. Elsethagen, L. Sun, V. Gurumoorthi, J. Chase, J. Li, and T. L. Windus, J. Chem. Inf. Model. 47, 1045 (2007).

${ }^{54}$ EPSRC National Service for Computational Chemistry Software, see http://www.nsccs.ac.uk 